\title{
Clinical factors affecting left ventricular end-diastolic pressure in patients with acute ST-segment elevation myocardial infarction
}

\author{
Xia Zhou, Mingming Lei, Donghui Zhou, Guoqing Li, Zhiying Duan, Shaobo Zhou, Yuanzhe Jin \\ Department of Cardiology, the 4th Affiliated Hospital of China Medical University, Shenyang, China \\ Contributions: (I) Conception and design: X Zhou, Y Jin; (II) Administrative support: Y Jin; (III) Provision of study materials or patients: All authors; \\ (IV) Collection and assembly of data: All authors; (V) Data analysis and interpretation: All authors; (VI) Manuscript writing: All authors; (VII) Final \\ approval of manuscript: All authors. \\ Correspondence to: Yuanzhe Jin. Department of Cardiology, the Fourth Affiliated Hospital of China Medical University, No. 4 Chongshan East Road, \\ Shenyang 110032, China. Email: c_club@126.com.
}

\begin{abstract}
Background: An association between left ventricular end-diastolic pressure (LVEDP) and outcomes of ischemic heart diseases has been reported. The present study aimed to investigate the LVEDP patterns and the effecting factors in patients with acute ST-segment elevation myocardial infarction (STEMI).

Methods: A total of 515 STEMI patients receiving immediate percutaneous coronary intervention (PCI) were divided into two groups according to their LVEDP before left ventricular angiography: LVEDP of $15 \mathrm{mmHg}$ or less (group A, n=145) and LVEDP above $15 \mathrm{mmHg}$ (group B, n=370). Blood samples were collected before and within 24 hours after PCI, and an ultrasonic cardiogram was conducted to measure left ventricular ejection fraction (EF\%) and to evaluate ventricular structure changes. The narrowness of each artery was measured with coronary angiography.
\end{abstract}

Results: In comparison with group A, patients in group B had a more infarction-related artery (IRA) descending branch and regional wall motion abnormality, a larger left atrial end-diastolic diameter (LAEDd) and a left ventricular end-diastolic diameter (LVEDd), a smaller EF\%, a higher level of myocardial necrosis markers, and a higher heart failure rate. Furthermore, LVEDP level was found to be positively correlated with Gensini score, LAEDd, LVEDd, N-terminal pro b-type natriuretic peptide, troponin T, uric acid, creatine kinase (CK), CK myocardial band, low-density lipoprotein cholesterol and fasting blood glucose, and negatively correlated with glomerular filtration rate and $\mathrm{EF} \%$.

Conclusions: LVEDP elevation has a higher incidence of heart failure and a higher risk of death, which is associated with the criminal blood vessels.

Keywords: Left ventricular end-diastolic pressure (LVEDP); ST-segment elevation; acute myocardial infarction; ventricular diastolic function; infarction related artery distribution; percutaneous coronary intervention (PCI)

Submitted Dec 23, 2019. Accepted for publication Feb 27, 2020.

doi: 10.21037/apm.2020.03.22

View this article at: http://dx.doi.org/10.21037/apm.2020.03.22

\section{Introduction}

The left ventricular dysfunction (LVD) causes secondary elevation of pulmonary pressure, which contributes to the more detrimental prognosis of myocardial infarction (MI) $(1,2)$. Previous studies have reported that ventricular diastolic dysfunction accompanied by elevated left ventricular diastolic pressure (LVDP) is common after acute ischemic infarction (3). Left ventricular end-diastolic pressure (LVEDP) has been used as an important marker of LVD in hemodynamic assessment $(4,5)$ and a strong predictor of prognosis in patients with ischemic heart diseases (6). Higher LVEDP has been associated with worse outcomes after balloon aortic valvuloplasty (7). Although noninvasive methods such as Doppler echocardiogram have been adopted to estimate LVEDP, catheterization remains 
the gold standard for its measurement. Therefore, in order to prevent adverse cardiac events, it should be beneficial to determine the predictive value of LVEDP and identify the factors that affect LVEDP, thus enabling prognostic prediction in patients with ischemic heart diseases.

Acute ST-segment elevation myocardial infarction (STEMI) is distinguishable from non-STEMI with respect to mechanisms, clinical symptoms, and prognosis. The current study specifically investigated factors that affect LVEDP in STEMI patients. The 1998 European Society of Cardiology work report (8) and its 2007 revised report (9) have stated that LVEDP above $16 \mathrm{mmHg}$ serves as a marker of $\mathrm{LV}$ diastolic dysfunction. Therefore, in the current study, an LVEDP of $15 \mathrm{mmHg}$, measured by left heart catheterization, was used as the cutoff value for investigating factors associated with elevated LVEDP.

\section{Methods}

\section{Patients}

STEMI patients admitted to the emergency room (ER) of the Fourth Affiliated Hospital of China Medical University (Shenyang, China) between January of 2014 and July of 2017 were included in this study. STEMI was diagnosed based upon a combination of increased MI biomarkers, clinical symptoms, and electrocardiography (ECG). Patients exhibiting acute pulmonary edema, shock, cerebral vascular disease, or severe impaired liver/renal functions, or whose MI onset was longer than 12 hours prior to enrollment were excluded. The study was approved by institutional ethics committee of the 4th Affiliated Hospital of China Medical University (No. EC-2017-KS-043) and informed consent was taken from all the patients. Written informed consent was obtained from the patient for publication of this study and any accompanying images. A copy of the written consent is available for review by the Editor-in-Chief of this journal. Patients were divided into two groups according to their pre-angiography LVEDP: group A (LVEDP of $15 \mathrm{mmHg}$ or less) and group B (LVEDP above $15 \mathrm{mmHg}$ ). All patients received percutaneous coronary intervention (PCI) within 12 hours after MI onset.

\section{Outcome assessment}

Demographic data were collected at patient admission to the hospital. Baseline cardiac function data and imaging data were obtained within 12 hours after the admission and before PCI. The endpoint of study was clinical death.

\section{Laboratory measurements}

A fasting blood sample was collected before PCI in order to conduct renal function assessment and routine blood examination with an AU640 automatic biochemistry analyzer (OLYMPUS, Inc., Tokyo, Japan) and an HL-750 automatic hematology analyzer (Beckman Coulter, Inc., USA), respectively. Additionally, a fasting blood sample was collected within 24 hours after PCI to measure the following parameters: triglyceride (TG), total cholesterol (TC), low-density lipoprotein (LDL) cholesterol, highdensity lipoprotein (HDL) cholesterol, fasting blood glucose (FBG), albumin, creatinine (Cr) and uric acid (UA). Serum troponin $\mathrm{T}(\mathrm{Tn} \mathrm{T})$, creatine kinase $(\mathrm{CK})$ and creatine kinase myocardial band (CKMB) were measured at 4, 8 , $16,24,32$, and 48 hours after MI onset, and the maximum value obtained during this series of measurements was used for analysis. $\mathrm{N}$-terminal pro b-type natriuretic peptide (NT-proBNP) level within the first 24 hours after PCI was measured by using electrochemiluminescent immunoassay. Glomerular filtration rate (GFR) was deduced from serum $\mathrm{Cr}$ value, after adjusting for age and gender.

Ultrasonic cardiogram and ejection function were assessed within 12 hours after admission to measure left ventricular end-diastolic diameter (LVEDd), left atrial end-diastolic diameter (LAEDd), left ventricular ejection fraction $(\mathrm{EF} \%)$, and also, to detect ventricular regional wall motion abnormalities.

To obtain a Gensini score for coronary artery disease, the narrowness of each segment was evaluated by three experienced technicians. It was calculated according to Gensini et al., and this score was then used to determine the severity of coronary disease.

LVEDP was obtained by averaging three recorded maximum pressures at the end of the deep exhalation, measured with a $5 \mathrm{~F}$ pigtail tube immediately before and after left ventricular angiography. The angiography applied $30 \mathrm{~mL}$ radio-contrast dye, at $15 \mathrm{~mL} / \mathrm{s}$ and $800 \mathrm{kPa}$. The difference between two LVEDPs was calculated as $\triangle$ LVEDP.

\section{Statistics}

SPSS version 19.0 (IBM Corp., Armonk, NY, USA) was used for data analysis. Continuous data were expressed as mean \pm standard deviation $(\bar{x} \pm \sigma)$. Chi-square test was used 
for categorical data comparison and an independent $t$-test for continuous data comparison. A Pearson test was used to analyze the correlation of LVEDP with coronary Gensini score, LAEDd, LVEDd, NT-proBNP, TnT, GFR, UA, CKMB, CK, LDL and FBG. One-way ANOVA was applied to compare LVEDP of different infarction-related arteries (IRAs). Comparison of the two groups was conducted using the Least Significant Difference (LSD) method. A P value of less than 0.05 was regarded as statistically significant.

\section{Results}

\section{Baseline data}

A total of 515 STEMI patients were enrolled and 370 (71.8\%) had an LVEDP greater than $15 \mathrm{mmHg}$ (group B). Baseline data are summarized in Table 1. In group A, the average hospital stay was 6.5 days, 30 cases had heart failure (Killip grade II+) (30/145, 20.7\%), and 2 cases died $(2 / 145,1.4 \%)$. In group B, the average hospital stay was 7.8 days, 114 cases had heart failure (Killip grade II+) (114/370, 30.8\%), and 9 cases died (9/370, 2.4\%). The two groups exhibited no significant difference in age, gender, smoking status, or concomitant pathologies. Laboratory parameters including TG, HDL, albumin, NT-proBNP and GFR and right ventricular size were similar between the two groups. However, groups A and B showed significant differences in Gensini score, Killip grading, infarct related arteries (IRA) composition, regional wall motion abnormality and the following laboratory parameters: TC, LDL, FBG, hemoglobin (Hbg), UA, CK, CKMB and TnT. In comparison with group A, group B also had higher LAEDd and LVEDd, and smaller EF\%. Moreover, patients in group B also exhibited higher levels of myocardial necrosis markers and higher heart failure.

\section{Changes in LVEDP and IRA}

LVEDP was significantly increased after angiography (Table 2). As for IRAs, LVEDP was significantly higher in the anterior descending branches than in the left circumflex and right coronary arteries, while $\triangle$ LVEDP was the highest in the right coronary artery and was significantly higher than that in anterior descending branches and left circumflex arteries (Table 2).

\section{Correlation of LVEDP and multiple parameters}

Pearson correlation analysis showed that LVEDP was positively correlated with Gensini score, LAEDd, LVEDd, NT-proBNP, TnT, GFR, UA, CKMB, CK, LDL and FBG, and negatively correlated with GFR and EF\%. Particularly, Gensini score, TnT, CKMB, CK and EF were highly correlated with LVEDP (in all instances, $\mathrm{P}<0.001$ ) (Table 3).

\section{Discussion}

In present study, the proportion of anterior descending branch in IRA of group B was significantly higher than that of group A. In comparison with group A, group B also showed a greater LVEDP change in the right coronary IRA before and after angiography and a higher incidence of heart failure.

In this study, LVEDP was positively correlated with TnT, CKMA, and CK. This result can be explained as impaired ventricular diastolic functioning resulting from MI elevates LVEDP.

Studies by Kirtane et al. (10-12) showed a correlation between elevated LVEDP, longer hospital stay and higher rate of heart failure within 30 days after a STEMI. Patients with an LVEDP greater than $24 \mathrm{mmHg}$ had a worse prognosis and a higher incidence of mortality. These studies suggested LVEDP as an independent predictor of re-hospitalization and prognosis after STEMI (13-15). Satiroglu et al. (16) identified STEMI as the cause of decreased left ventricular compliance, increased LVEDP, and impaired left ventricular diastolic function. LVEDP can be measured before and after PCI without any resulting complications, and therefore can be used for quick evaluation of diastolic function improvements.

Acute MI is an acute myocardial ischemic necrosis that often results from complete coronary stenosis due to a thrombosis induced by broken unstable plaques, and it is a common and crisis condition of left heart diseases (17). In the current study, severe coronary pathology was initially verified by pre-angiography catheterization in STEMI patients; $71.8 \%$ patients had an LVEDP above $15 \mathrm{mmHg}$ before PCI suggests that most STEMI patients have ventricular systolic and/or diastolic dysfunction at the early stage. If systolic or diastolic dysfunction continues, the left ventricular filling pressure and left atrial pressure will increase, leading to obstructed pulmonary intravenous circulation and increased pulmonary pressure with or without pathological changes in small arteries (18).

A few studies have reported that LDL, FBG and UA are independent risk factors for cardiovascular diseases and are closely associated with the severity and prognosis of 
Table 1 Baseline data

\begin{tabular}{|c|c|c|c|}
\hline Subjects & Group A (n=145) & Group B $(n=370)$ & $P$ value \\
\hline Gender (male/female) & $114 / 31$ & $269 / 101$ & 0.167 \\
\hline Smoking, n (\%) & $112(77.2)$ & $293(79.2)$ & 0.628 \\
\hline Killip grading, $n(\%)$ & & & 0.022 \\
\hline \multicolumn{4}{|l|}{ Concomitant diseases, n (\%) } \\
\hline Old myocardial infarction (\%) & $10(6.9)$ & $25(6.8)$ & 0.955 \\
\hline Hypertension (\%) & $76(52.4)$ & $206(55.7)$ & 0.504 \\
\hline Diabetes (\%) & $25(17.2)$ & $90(24.3)$ & 0.083 \\
\hline $\mathrm{TC}(\mathrm{mmol} / \mathrm{L})$ & $4.32 \pm 0.75$ & $4.59 \pm 0.89$ & 0.001 \\
\hline $\mathrm{TG}(\mathrm{mmol} / \mathrm{L})$ & $1.73 \pm 1.68$ & $1.54 \pm 0.91$ & 0.180 \\
\hline LDL (mmol/L) & $2.75 \pm 0.63$ & $3.12 \pm 0.82$ & $<0.001$ \\
\hline $\mathrm{HDL}(\mathrm{mmol} / \mathrm{L})$ & $1.00 \pm 0.29$ & $0.97 \pm 0.22$ & 0.276 \\
\hline FBG (mmol/L) & $6.53 \pm 1.46$ & $7.55 \pm 3.02$ & $<0.001$ \\
\hline $\mathrm{Hbg}(\mathrm{g} / \mathrm{L})$ & $136.93 \pm 11.03$ & $134.26 \pm 16.52$ & 0.034 \\
\hline Albumin (g/L) & $38.31 \pm 3.22$ & $38.50 \pm 3.16$ & 0.550 \\
\hline NT-proBNP (pg/mL) & $1,535.62 \pm 1,412.46$ & $1,831.58 \pm 2,045.28$ & 0.123 \\
\hline \multicolumn{4}{|l|}{ Ultrasonic cardiogram parameters } \\
\hline LAEDd $(\mathrm{mm})$, mean \pm SD & $36.21 \pm 3.22$ & $37.19 \pm 3.38$ & 0.003 \\
\hline LVEDd $(\mathrm{mm})$, mean \pm SD & $47.62 \pm 4.01$ & $49.99 \pm 4.81$ & $<0.001$ \\
\hline $\mathrm{RV}(\mathrm{mm})$, mean $\pm \mathrm{SD}$ & $18.96 \pm 1.59$ & $19.24 \pm 1.82$ & 0.084 \\
\hline $\mathrm{EF} \%$, mean $\pm \mathrm{SD}$ & $61.28 \pm 5.78$ & $57.43 \pm 7.53$ & $<0.001$ \\
\hline Regional wall motion abnormality, $\mathrm{n}(\%)$ & $64(44.1)$ & $236(63.8)$ & $<0.001$ \\
\hline \multicolumn{4}{|l|}{ Coronary disease evaluation } \\
\hline Time to IRA cleaning (hour), mean \pm SD & $5.83 \pm 3.06$ & $5.45 \pm 2.51$ & 0.183 \\
\hline Gensini score, mean \pm SD & $43.43 \pm 22.60$ & $59.05 \pm 26.62$ & $<0.001$ \\
\hline $\begin{array}{l}\text { IRA composition: anterior descending branch/ } \\
\text { circumflex/right coronary artery, } \mathrm{n}(\%)\end{array}$ & 49/15/81 (33.8/10.3/55.9) & 202/34/134 (54.6/9.2/36.2) & $<0.001$ \\
\hline
\end{tabular}

SD, standard deviation; TC, total cholesterol; TG, triglyceride; LDL, low-density lipoprotein; HDL, high-density lipoprotein; FBG, fasting blood glucose; Hbg, hemoglobin; NT-proBNP, N-terminal pro b-type natriuretic peptide; TnT, troponin T; CKMB, creatine kinase myocardial band; CK, creatine kinase; GFR, glomerular filtration rate; UA, uric acid; LAEDd, left atrial end-diastolic diameter; LVEDd, left ventricular end-diastolic diameter; RV, right ventricle; EF\%, ejection fraction; IRA, infarction-related artery. 
Table 2 LVEDP changes

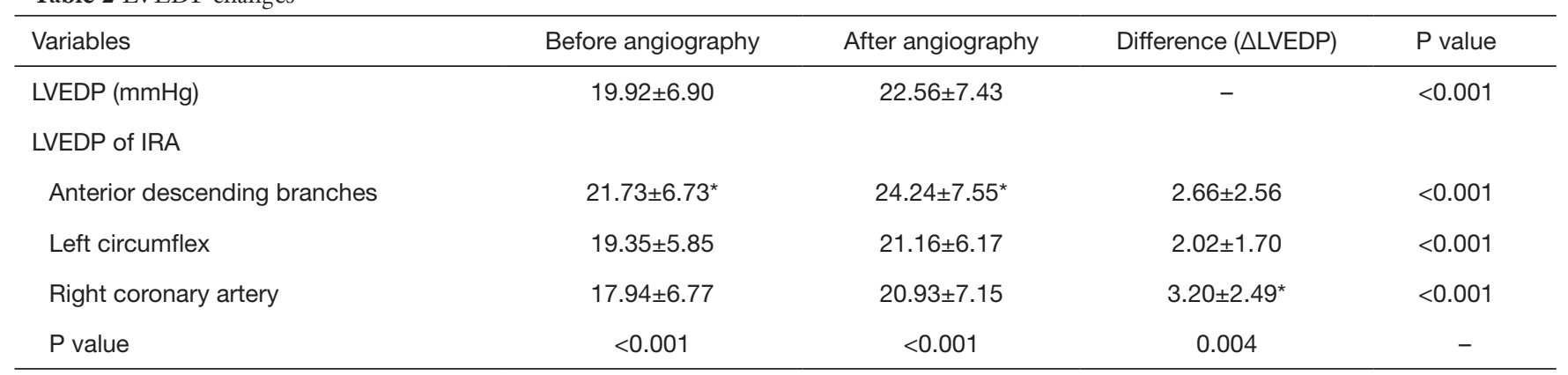

*, statistical differences between the groups. LVEDP, left ventricular end-diastolic pressure; IRA, infarction-related artery.

Table 3 Correlation of LVEDP and clinical parameters

\begin{tabular}{lcc}
\hline Variables & $r$ & $P$ value \\
\hline Coronary Gensini score & 0.289 & $<0.001$ \\
EF\% & -0.287 & $<0.001$ \\
LAEDd & 0.136 & 0.003 \\
LVEDd & 0.213 & $<0.001$ \\
NT-proBNP & 0.154 & 0.001 \\
TnT & 0.332 & $<0.001$ \\
GFR & -0.100 & 0.023 \\
UA & 0.136 & 0.002 \\
CKMB & 0.303 & $<0.001$ \\
CK & 0.376 & $<0.001$ \\
LDL & 0.163 & $<0.001$ \\
FBG & 0.206 & $<0.001$ \\
\hline
\end{tabular}

LVEDP, left ventricular end-diastolic pressure; EF\%, ejection fraction; LAEDd, left atrial end-diastolic diameter; LVEDd, left ventricular end-diastolic diameter; NT-proBNP, N-terminal pro b-type natriuretic peptide; TnT, troponin T; GFR, glomerular filtration rate; UA, uric acid; CKMB, creatine kinase myocardial band; CK, creatine kinase; LDL, low-density lipoprotein; FBG, fasting blood glucose.

coronary diseases and heart failure (19-22). Results of this study showed that levels of LDL, TC, FBG and UA were significantly higher in the LVEDP elevation group; LDL, FBG, UA and NT-proBNP were positively, and GFR was negatively correlated with LVEDP. LVEDP also increased with impaired renal function. These data suggested that LVEDP can be used to predict the severity and prognosis of coronary diseases.

Gensini score quantifies the severity of coronary disease; a higher score indicates more severe disease and impaired systolic and diastolic functions (23). In this study, LVEDP was positively correlated with Gensini score, thus suggesting that LVEDP is correlated with the severity of coronary disease.

When the LVEDP is $15 \mathrm{mmHg}$ or less (group A), IRAs are most frequently right coronary arteries, followed by anterior descending branches; in contrast, when the LVEDP is above $15 \mathrm{mmHg}$ (group B), IRAs are most frequently anterior descending branches followed by right coronary arteries. These data suggested that LVEDP elevation is more likely in STEMI patients who exhibit more infarctionrelated pathology involving anterior descending branches. Hence, pathological changes in anterior descending branches appear to have a greater impact on LVEDP. In all cases, LVEDP increased after left ventricular angiography; patients showing infarction-related pathology in the right coronary artery as the largest proportion of IRAs had the greatest increase in LVEDP. This may be explained as follows: the right ventricular function is impaired because of right coronary artery disease, increased right heart filling pressure, and left ventricular septum, leading to restricted left ventricular dilation, which exacerbates the increase in left ventricular workload after ventricular angiography and the consequent increase in left ventricular filling pressure.

Results showed that the rate of regional wall motion abnormality, LAEDd, LVEDd and EF\% were significantly different in the two groups. LAEDd and LVEDd were higher in group $\mathrm{B}$ while $\mathrm{EF} \%$ was lower in this group. The LVEDP elevation group included a larger proportion of patients with regional wall motion abnormality, as well as more patients with heart failure. One possible mechanism underlying these observations is that the myocardial necrosis after STEMI leads to a fibrosis scar and myocardial remodeling, resulting in increases of LAEDd and LVEDd, which in turn lead to the LVD and increased LVEDP. As 
LVEDP continuously increases, the pulmonary artery pressure increases because of the escape beat arrhythmia, ultimately leading to heart failure.

In many studies, left ventricular diastolic function were evaluated by either Doppler echocardiography or pulmonary capillary wedge pressure, which are indirect methods impacted by many unstable factors, and therefore, cannot accurately reflect diastolic functions $(1-3,24)$. Catheterization remains the standard method to measure LVEDP. This study measured LVEDP during PCI, thus providing reliable data to evaluate left ventricular diastolic functions.

This study has the following limitations. First, this is a single-center study with a small sample size of local patients, and therefore, selection bias could not be eliminated. Second, the study only recorded clinical parameters prior and subsequent to PCI; hence, follow-up data for these patients are not available. Multi-center studies with larger sample sizes would better elucidate LVEDP changes in STEMI patients.

\section{Acknowledgments}

Funding: This study was supported by Natural science fund project in liaoning province (Project number: 2015020486).

\section{Footnote}

Conflicts of Interest: All authors have completed the ICMJE uniform disclosure form (available at http://dx.doi. org/10.21037/apm.2020.03.22). The authors have no conflicts of interest to declare.

Ethical Statement: The authors are accountable for all aspects of the work in ensuring that questions related to the accuracy or integrity of any part of the work are appropriately investigated and resolved. The study was conducted in accordance with the Declaration of Helsinki (as revised in 2013). The study was approved by institutional ethics committee of the 4th Affiliated Hospital of China Medical University (No. EC-2017-KS-043) and informed consent was taken from all the patients.

Open Access Statement: This is an Open Access article distributed in accordance with the Creative Commons Attribution-NonCommercial-NoDerivs 4.0 International License (CC BY-NC-ND 4.0), which permits the noncommercial replication and distribution of the article with the strict proviso that no changes or edits are made and the original work is properly cited (including links to both the formal publication through the relevant DOI and the license). See: https://creativecommons.org/licenses/by-nc-nd/4.0/.

\section{References}

1. Miller WL, Mahoney DW, Michelena HI, et al. Contribution of ventricular diastolic dysfunction to pulmonary hypertension complicating chronic systolic heart failure. JACC Cardiovasc Imaging 2011;4:946-54.

2. Møller JE, Hillis GS, Oh JK, et al. Prognostic importance of secondary pulmonary hypertension after acute myocardial infarction. Am J Cardiol 2005;96:199-203.

3. Møller JE, Sondergaard E, Poulsen SH, et al. Pseudonormal and restrictive filling patterns predict left ventricular dilation and cardiac death after a first myocardial infarction: a serial color M-mode Doppler echocardiographic study. J Am Coll Cardiol 2000;36:1841-6.

4. Salem R, Denault AY, Couture P, et al. Left ventricular end-diastolic pressure is a predictor of mortality in cardiac surgery independently of left ventricular ejection fraction. Br J Anaesth 2006;97:292-7.

5. Ahmed I, House CM, Nelson WB. Predictors of inotrope use in patients undergoing concomitant coronary artery bypass graft (CABG) and aortic valve replacement (AVR) surgeries at separation from cardiopulmonary bypass (CPB). J Cardiothorac Surg 2009;4:24.

6. Li YY, Bush CA, Orsini A, et al. Predictors of inpatient outcomes in hospitalized patients after left heart catheterization. Am J Cardiol 2009;103:486-90.

7. Cubeddu RJ, Don CW, Horvath SA, et al. Left ventricular end-diastolic pressure as an independent predictor of outcome during balloon aortic valvuloplasty. Catheter Cardiovasc Interv 2014;83:782-8.

8. How to diagnose diastolic heart failure. European Study Group on Diastolic Heart Failure. Eur Heart J 1998;19:990-1003.

9. Paulus WJ, Tschope C, Sanderson JE, et al. How to diagnose diastolic heart failure: a consensus statement on the diagnosis of heart failure with normal left ventricular ejection fraction by the Heart Failure and Echocardiography Associations of the European Society of Cardiology. Eur Heart J 2007;28:2539-50.

10. Kirtane AJ, Bui A, Murphy SA, et al. Association of epicardial and tissue-level reperfusion with left ventricular end-diastolic pressures in ST-elevation myocardial 
infarction. J Thromb Thrombolysis 2004;17:177-84.

11. Planer D, Mehran R, Witzenbichler B, et al. Prognostic utility of left ventricular end-diastolic pressure in patients with ST-segment elevation myocardial infarction undergoing primary percutaneous coronary intervention. Am J Cardiol 2011;108:1068-74.

12. Bagai A, Armstrong PW, Stebbins A, et al. Prognostic implications of left ventricular end-diastolic pressure during primary percutaneous coronary intervention for ST-segment elevation myocardial infarction: Findings from the Assessment of Pexelizumab in Acute Myocardial Infarction study. Am Heart J 2013;166:913-9.

13. Ndrepepa G, Cassese S, Hashorva D, et al. Relationship of left ventricular end-diastolic pressure with extent of myocardial ischemia, myocardial salvage and long-term outcome in patients with ST-segment elevation myocardial infarction. Catheter Cardiovasc Interv 2019;93:901-9.

14. Brienesse SC, Davies AJ, Khan A, et al. Prognostic Value of LVEDP in Acute Myocardial Infarction: a Systematic Review and Meta-Analysis. J Cardiovasc Transl Res 2018;11:33-5.

15. Saito D, Nakanishi R, Watanabe I, et al. Combined assessment of left ventricular end-diastolic pressure and ejection fraction by left ventriculography predicts longterm outcomes of patients with ST-segment elevation myocardial infarction. Heart Vessels 2018;33:453-61.

16. Satıroğlu O, Cicek Y, Bostan M, et al. Acute change in left ventricle end-diastolic pressure after primary percutaneous coronary intervention in patients with ST segment elevation myocardial infarction. Am Heart Hosp J 2010;8:E86-90.

17. Task Force on the management of ST-segment elevation acute myocardial infarction of the European Society

Cite this article as: Zhou X, Lei M, Zhou D, Li G, Duan Z, Zhou S, Jin Y. Clinical factors affecting left ventricular enddiastolic pressure in patients with acute ST-segment elevation myocardial infarction. Ann Palliat Med 2020;9(4):1834-1840. doi: 10.21037/apm.2020.03.22 of Cardiology (ESC), Steg PG, James SK, et al. ESC Guidelines for the management of acute myocardial infarction in patients presenting with ST-segment elevation. Eur Heart J 2012;33:2569-619.

18. Galiè N, Hoeper MM, Humbert M, et al. Guidelines for the diagnosis and treatment of pulmonary hypertension: the Task Force for the Diagnosis and Treatment of Pulmonary Hypertension of the European Society of Cardiology (ESC) and the European Respiratory Society (ERS), endorsed by the International Society of Heart and Lung Transplantation (ISHLT). Eur Heart J 2009;30:2493-537.

19. Brodov Y, Chouraqui P, Goldenberg I, et al. Serum uric acid for risk stratification of patients with coronary artery disease. Cardiology 2009;114:300-5.

20. Iwakura K, Ito H, Ikushima M, et al. Association between hyperglycemia and the no-reflow phenomenon in patients with acute myocardial infarction. J Am Coll Cardiol 2003;41:1-7.

21. Pitt B, Loscalzo J, Ycas J, et al. Lipid levels after acute coronary syndromes. J Am Coll Cardiol 2008;51:1440-5.

22. Bartnik M, Ryden L, Ferrari R, et al. The prevalence of abnormal glucose regulation in patients with coronary artery disease across Europe. The Euro Heart Survey on diabetes and the heart. Eur Heart J 2004;25:1880-90.

23. Gensini GG. A more meaningful scoring system for determining the severity of coronary heart disease. Am J Cardiol 1983;51:606.

24. Mutlak D, Lessick J, Carasso S, et al. Utility of pulmonary hypertension for the prediction of heart failure following acute myocardial infarction. Am J Cardiol 2012;109:1254-9. 